\title{
Semantic Turkey: A browser-integrated environment for knowledge acquisition and management
}

Editor: Oscar Corcho, Universidad Politécnica de Madrid (UPM), Spain

Solicited reviews: Roberto García, Universitat Pompeu Fabra (UPF), Spain; Leopold Sauermann, Gnowsis, Austria; Knud Möller, DERI Research Center, Galway, Ireland

\author{
Maria Teresa Pazienza ${ }^{\mathrm{a}}$, Noemi Scarpato ${ }^{\mathrm{b}}$, Armando Stellato $^{\mathrm{a},{ }^{*}}$ and Andrea Turbati ${ }^{\mathrm{b}}$ \\ ${ }^{a}$ University of Tor Vergata, Dept. of Enterprise Engineering, Via del Politecnico 1, Rome, 00133, Italy \\ ${ }^{\mathrm{b}}$ University of Rome, Tor Vergata, Dept. of Computer Science, Systems and Production, Via del Politecnico 1, \\ Rome, 00133, Italy
}

\begin{abstract}
Born four years ago as a Semantic Web extension for the web browser Firefox, Semantic Turkey pushed forward the traditional concept of links\&folders-based bookmarking to a new dimension, allowing users to keep track of relevant information from visited web sites and to organize the collected content according to standard or personally defined ontologies. Today, the tool has broken the boundaries of its original intents and can be considered, under every aspect, an extensible platform for knowledge management and acquisition. The semantic bookmarking and annotation facilities of Semantic Turkey are now supporting just a part of a whole methodology where different actors, from domain experts to knowledge engineers, can cooperate in developing, building and populating ontologies while navigating the Web.
\end{abstract}

Keywords: Semantic browsing, semantic annotation, semantic bookmarking, ontology development

\section{Introduction}

The Semantic Web is becoming ever and ever a concrete reality: with SPARQL reaching W3C recommendation in 2008 [1], languages for data representation and querying have finally completed standardization, closing the gap on usability of Semantic Web technologies in real case scenarios. At the same time, initiatives such as Linked Open Data [2] have boosted the process of data provisioning on the Web.

Finally, interests and research in SW technologies have definitely migrated from mere ontology development (which has now met industry standards) to the discovery and provision of applications which can exploit full Semantic Web potential: homogenous access to distributed information providers, connecting conceptual and information ${ }^{1}$ resources on the Web of Open Data.

\footnotetext{
* Corresponding author. E-mail: stellato@info.uniroma2.it.
}

With this scenario in mind, we have worked towards the definition of a Semantic Web browser extension which is two-fold in its offer: first, it is of interest for ontology developers and domain experts (since it aims at facilitating the process of knowledge acquisition and development even for non technology-savvy users); second, it provides an extensible infrastructure over which SW applications, needing and relying on rock-solid web browsing functionalities as well as on RDF management capacities, can be developed and deployed. These objectives have been pursued during a two-year period of finalization and reengineering of Semantic Turkey [3], a Semantic Web extension for the popular Firefox $^{2}$ web browser.

\footnotetext{
${ }^{1}$ Following from the definition of information/non-information resources given in: http://www.w3.org/2001/tag/doc/httpRange-14/ 2007-05-31/HttpRange-14.

${ }^{2}$ http://www.mozilla.com/en-US/firefox/
} 
In this paper, we describe the original version of this application as it was conceived in the beginning, and introduce and discuss the main innovations which transformed the new incarnation of Semantic Turkey into an open and extensible platform for Semantic Web development.

\section{Related work}

Due to the multifaceted nature of our platform, an overview of related research should embrace diverse fields such as ontology editing and visualization, Semantic Web browsing, (social/semantic) bookmarking solutions and semantic annotation. In this section we recall the main works in these areas, and provide insight readings for a thorough view.

\subsection{Ontology editing tools}

Probably the most used and widely known ontology editing platform is Protégé $[4,5]$. Realized at the Center for Biomedical Informatics Research of the University of Stanford, Protégé has been for years the leading environment for ontology management and has also contributed to the first spread of Semantic Web Technologies in research communities and industries. The Protégé project is currently active, with the Stanford team carrying on development and maintenance of Protégé 3.x, and the University of Manchester developing the next version: Protégé 4.x, which is still in beta development. Another interesting framework is offered by the Neon toolkit [6]: an extensible ontology engineering environment, which has been developed inside the homonymous integrated project co-funded by the European Commission's Sixth Framework Programme. Today, ontology development has reached industry standard, as witnessed by commercial off-the-shelf products such as Topbraid Composer ${ }^{3}$.

\subsection{Information visualization/semantic browsing}

Regarding information visualization through Semantic Web technologies, or "semantic browsing", the first reference which comes to mind is probably the Haystack web client [7]. Developed at the MIT laboratories, it was conceived as an application that could be used to browse arbitrary Semantic Web information in much the same fashion as a web browser

\footnotetext{
${ }^{3}$ http://topbraidcomposer.info/
}

can be used to navigate the Web. Standard point-andclick semantics let Haystack user navigate over aggregation of data projected from RDF repositories available from different arbitrary locations. The application has been built as an extension for the popular IDE Eclipse ${ }^{4}$; this choice facilitates extension of the tool thanks to Eclipse's flexible plug-in mechanism, but requires the user to adopt its framework as a platform for browsing the Web and collecting data from it: a negative impact for the average user, who would just prefer to rely on their trusted personal web browser and try out other features which are not too invasive for their usual way of working. An opposite approach is being followed by Magpie [8], which is deployed as a plug-in for the Microsoft Internet Explorer Web Browser. In its first incarnation, Magpie allowed for semantic browsing, intended as the parallel navigation of traditional web content and of its associated semantic layer (an ontology associated to the web resource, which semantically describes its content). Magpie also allows for collaborative semantic web browsing, in that different persons may gather information from the same web resource and exchange it on the basis of a common ontology. Later work on Magpie [9] extended the platform more and more towards the vision of the Semantic Web as "an open Web of interoperable applications" [10], by allowing bi-directional exchange of information among users and services, which can be opportunistically located and composed, either manually (web services) or automatically (semantic web services).

From some of the same authors as those of Haystack, comes Piggy-Bank [11], an extension for the Firefox web browser that lets Web users extract individual information items from within web pages and save them in RDF, replete with metadata. Piggy Bank then lets users make use of these items right inside the same web browser. These items, collected from different sites, can then be browsed, searched, sorted, and organized, regardless of their origins and types. Piggy-Bank users may also rely on Semantic Bank, a web server application that lets them share the Semantic Web information they have collected, enabling - as for Magpie - collaborative efforts to build sophisticated Semantic Web information repositories from daily navigation through their enhanced web browser. Finally, the father of the WWW shared his perspective on what a Data Browser should be with Tabulator [12]. Tabulator focuses on pioneering aspects related to navigation of linked open data, by following dereferenced URIs across their ontology

\footnotetext{
${ }^{4}$ http://www.eclipse.org/
} 
definition sparse in the web of data, and being able to extract data from heterogeneous documents whenever these expose some GRDDL declaration for data extraction through document transformation. As for Semantic Turkey, Tabulator does not offer dedicated UIs focused on a given domain (with the sole exception of geo-spatial and temporal coordinates inspection through calendar/timeline and maps views respectively), but rather presents itself as a domainagnostic data browsing tool. Also, for SPARQL it allows both a generic SPARQL query tool and a basic query-by-example wizard.

\subsection{Semantic/social annotation/bookmarking}

The most popular "social bookmarking" service, del.icio.us ${ }^{5}$, is a service for building personal collections of bookmarks and access them online. It is possible, through the same service, to add links to a collection of bookmarks, to categorize the related sites with keywords, and to share the personal collection with other users. Regarding semantic annotation, research in this field is mainly addressing three aspects: how to set up an annotation environment, how to improve the process and extend to several media, and how to automate it. The Annotea W3C project [13], suggests RDF based standards for representation of annotations, and provides a general architecture for establishing client-server annotation frameworks. Several clients have been developed for this architecture, such as Amaya [14] and Annozilla [15]. Melita [16] and KIM [17] are probably the most prominent examples of applying decades of research on NLP to automate semantic annotation. AKTive Media [18], the successor of Melita, pushes forward the concept of annotation to cover different media other than text. A thorough overview on Semantic Annotation can be found in [19].

\section{History and motivations}

What lacks from the approaches above is a really integrated solution which is able to combine the best of all worlds from visualization, annotation and ontology development. Regarding annotation tools, as remarked in [19], though "there are signs that annotation systems are giving users more control of ontologies", still "ontology maintenance $[\ldots]$ is poorly sup-

\footnotetext{
${ }^{5}$ http://delicious.com/
}

ported, or not supported at all, by the current generation of [semantic annotation] tools".

Seen from the other side (ontology development tools), the RDF family (RDF, RDFS, OWL, SKOS) of models as well as many standard vocabularies such as Dublin Core, offer properties providing metaknowledge about what is behind the creation of resources in an ontology (such as the RDF rdfs:seeAlso, or Dublin Core dc:relation, dc:source and dc:subject). This is because the specification of a domain should be naturally connected with the process of acquiring knowledge from external sources, and thus of documenting references to them, to better qualify the nature of formalized concepts. However, ontology development tools seem to live in a world of mere algebraic representation, requiring lot of manual work or parallel use of different tools if different actors need to cooperate and make reference to existing information (re)sources.

It is our idea that access to and interaction with the greatest source of information available today (the Web), should be ideally integrated in tools for Knowledge Modeling and (in particular) Acquisition.

Semantic Turkey (ST from now on) differs from similar, previously described approaches, by mixing ontology development functionalities with the ease of use of a system for acquiring knowledge from the web. This way, instead of working on different frameworks and producing different kind of data which need to be integrated, domain experts may start to sketch ontologies and keep track of the information they get from the Web, leave comments and references which can then be reused and examined by knowledge engineers in continuous refinement circles.

\subsection{The origins}

Semantic Turkey had been initially developed as a prototype for a Web Browser extension with advanced bookmarking capabilities [20]: its mission was to go beyond the vague semantics (with respect to information organization) of traditional links\& folders bookmarking, and promote a new paradigm, aiming at "a clear separation between (acquired) knowledge data (the WHAT) and their associated information sources on the Web (the WHERE)". We thus gave meaning to Semantic Bookmarking as to indicate the process of eliciting information from (web) documents, to acquire new knowledge and represent it through knowledge representation standards, while keeping reference to the original infor- 


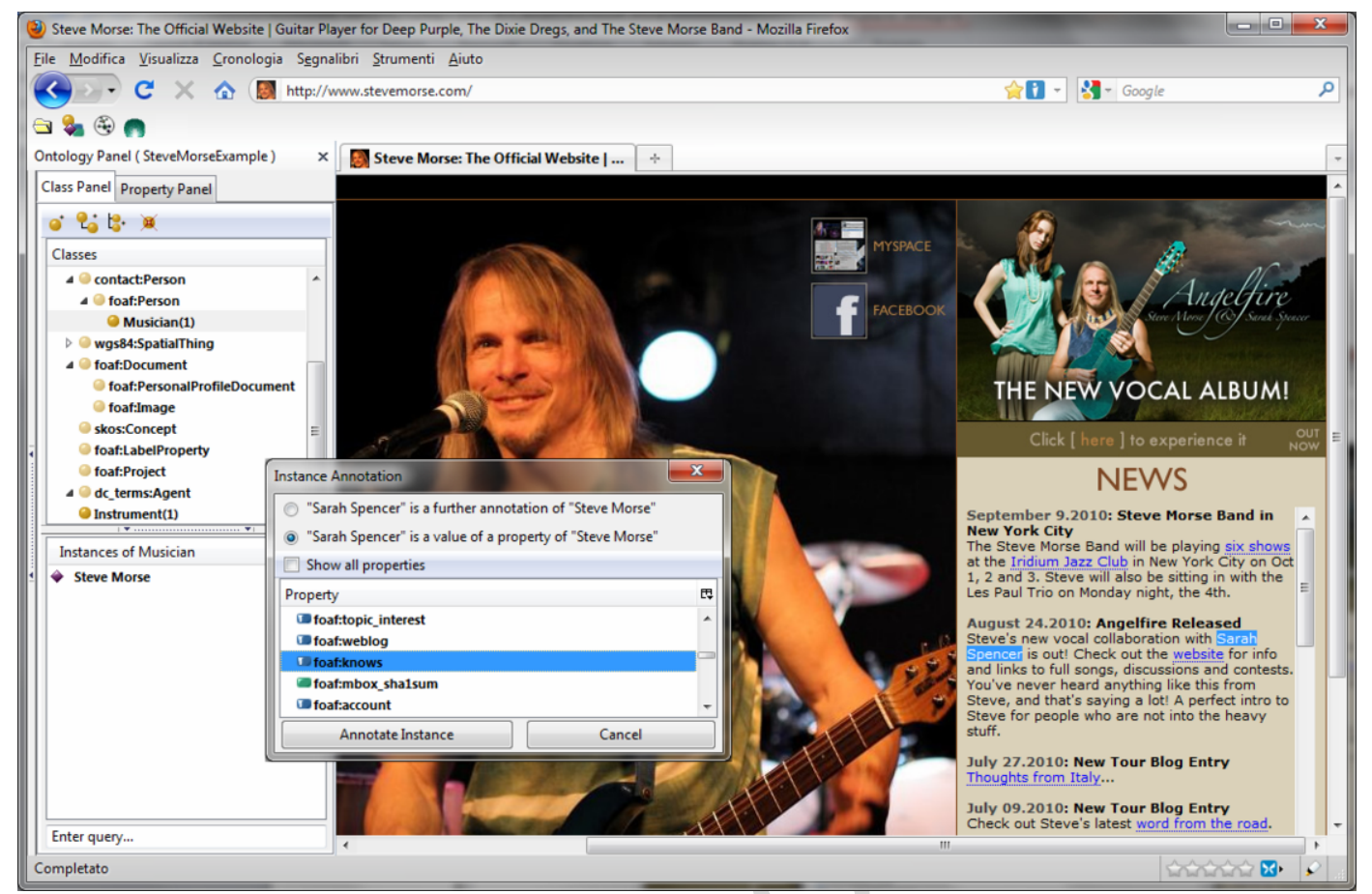

Fig. 1. Semantic bookmarking with Semantic Turkey.

mation sources ${ }^{6}$. The main difference with Semantic Annotation resides in the focus: the term "Semantic Annotation", though being subject (as underlined in [17]) to slightly different interpretations, which are in some cases too much bound to the specific research settings where the term has been adopted (e.g. in $[21,22]$ and, again, in [17]), has converged in literature towards the definition of "the process of associating portions of text of analyzed documents to predefined sets of semantic descriptors". So, the text is the focus of Semantic Annotation, whereas the first objective of Semantic Turkey was (and still is) to facilitate users in acquiring and organizing their knowledge, while keeping at the same time references to the source of information which are being consulted. Also, in a ever-changing setting as the WWW, keeping and maintaining precise reference (pointers to position in documents) to textual content would produce information doomed to corrupt, due to modifications of the bookmarked pages: for this reason, pointers to pages as a whole (i.e. bookmarks) were considered a good compromise for this task ${ }^{7}$. This

\footnotetext{
${ }^{6}$ This definition is aligned with the one of Social Semantic Bookmarking provided in [32], though the social aspects are not explored in this work.

${ }^{7}$ Though traditional Semantic Annotation is still made possible thanks to extensions thought for this, such as: http://semantic-
}

idea thus translated into a series of functionalities for the user, which, through very easy-to-use drag'n'drop gestures, could select textual information from web pages, create objects in a given domain and annotate their presence on the Web by keeping track of the selected text and of its provenance(web page $u r l$, title etc.). An example is given in Fig. 1 where the user is adding the musician Steve Morse as an object in their ontology, while at the same time decorates it with a bookmark to their homepage and provides further details about him (the instrument he plays, the musical genre etc.) getting them from that same page.

\subsection{From semantic bookmarking to knowledge management and acquisition}

Standing on the shouldersof mature results from research and development on Semantic Web technologies, such as Sesame [23], OWLim [24] and AllegroGraph ${ }^{8}$ as well as on a robust platform such as the Firefox web browser, Semantic Turkey differs from other existing approaches which are more spe-

turkey.uniroma2.it/extensions/rangeannotator/ which allows for precise reference to elements in the pages, through use of xpointers (http://www.w3.org/TR/xptr/).

${ }^{8} \mathrm{http}: / /$ www.franz.com/agraph/allegrograph/ 
cifically tailored towards knowledge management and editing[4], semantic mashup and browsing [9,11] and pure semantic annotation $[16,13]$ by introducing a new dimension which is unique to the process of building new knowledge while exploring the Web to acquire it.

By focusing on this aspect, we went beyond the original concept of semantic bookmarking and tried to amplify the potential of a complete knowledge management and acquisition system: we thus aimed at reducing the impedance mismatch between domain experts and knowledge investigators on the one side, and knowledge engineers on the other, providing them with a unifying platform for acquiring, building up, reorganizing and refining knowledge.

\subsection{Target users for Semantic Turkey}

Ontology editors are obviously tools thought for technology-savvy people: the whole range of editing possibilities offered by an editor fully-compliant with W3C vocabularies of the RDF family do not easily match up with the profile of an average librarian or domain expert. However, while Knowledge Engineers are expected to get the maximum return from such kind of tools, this does not mean that these cannot provide different levels and modalities of interaction for different users. In particular, with Semantic Turkey, domain experts can be easily instructed on how to add new concepts/properties to domain representations, on how to import external ontologies etc., while tasks which do not imply strong modeling skills, such as data entry, can be easily performed with almost no learning curve at all (and are also simplified by the acquire-through-annotation functionalities described in the next section). Finally, the extension possibilities of Semantic Turkey (see Sections 4.3 and 6.2) allow for unlimited customization of user interfaces (other than application logic) so that dedicated tools and system - thought for specific exigencies - can be built on top of the main platform.

\section{User interaction}

ST is now an open editor for data modeled upon languages of the RDF family, allowing the exploitation of almost all of those language potentialities (currently, it does not allow editing of complex OWL descriptions, though it loads them and reasoners exploit their content; also, SKOS and SKOS-XL sup- port is being provided as an experimental feature and will be finalized in the next release ${ }^{9}$ ).

Users can browse and edit (Fig. 2) the ontology by using ST like any other ontology editing (OE) tool. Unlike other ontology tools embedded in the web browser (such as Piggy-Bank [11]), which rely on web-based rendering of user interfaces, Semantic Turkey offers complete interaction with the ontology via the XUL interface completely integrated in the browser. The user is thus not diverted from web navigation (i.e. the main browser panel is still focused on the visited web page, which would otherwise be replaced by the HTML UI) and may, at the same time, maintain focus over both the observed web page and the ontology.

To allow maximum flexibility, every element in the ontology can now be added through the advanced bookmarking/annotation functionalities or directly through the ontology editor (in both cases, further annotations can be added later to the created objects).

Figure 3 shows the different annotation/knowledge acquisition possibilities offered by the functionalities based on integration with the hosting web browser: the process is multifaceted in its possible outcomes, though very easy to carry out, since it depends on implicit, contextual factors, such as where in the ontology the user drops the element dragged from the page, as well as on simple interaction steps with the user (like choosing if adding new annotations for a previous element or adding a value for a property, followed by further possibilities depending on the kind of property).

\section{1. “Macroing” series of ontology editing operations}

The drag'n'drop features for capturing data have been conceived to speed up the process of knowledge acquisition, allowing for complex series of ontology editing operations to be implicitly executed, depending on the specific action performed by the user. In the previous example, if we drag "Deep Purple" over the musician Steve Morse, and then select the playsInBand object property, the following update operations on the underlying ontology are performed:

- creation of an instance with local name "DeepPurple" (taken after the selected text), if it is a new resource not yet present in the ontology

\footnotetext{
${ }^{9}$ The version of Semantic Turkey referred in this work is 0.7.2.
} 


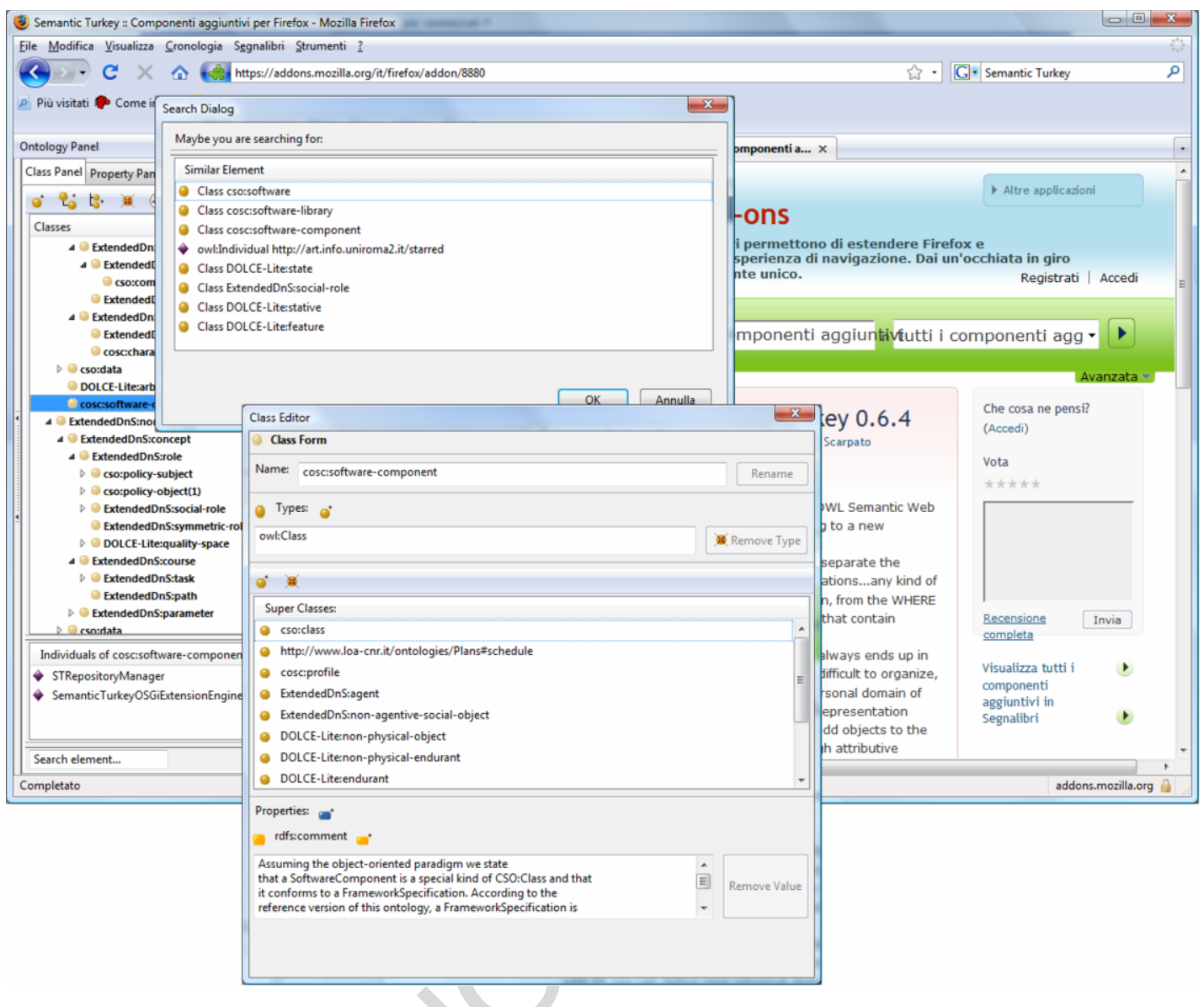

Fig. 2. Ontology browsing and editing.

- assertion of a relation (identified by the chosen object property) between the selected object (the "Deep Purple" band) and the instance where the text has been dropped (Steve Morse)

- the assertion of the rdf:type relation between the object of the above relation and the class selected from the range of the object property (e.g. DeepPurple as a MusicBand, or even a RockBand subclass, because the user is prompted with class-trees rooted on classes featured in the ranges of the selected object property)

- creation of the bookmarked page (as an ontology individual) and associated data (title, url etc.)

- creation of a semantic annotation linking the created individual to the bookmarked web page.

The cost for the above operations is just a drag\& drop and a couple of intuitive choices among those proposed through the acquisition process.

\subsection{Real "Open World Assumption" - Aware approach to user interface}

Whereas constraint-checking approaches to UI exploit constraints defined in the underlying data model as a strict base for populating form-filling panels, not allowing any operation which could invalidate the constraints, a tool whose knowledge model is based on the open world assumption and on inferential capabilities, uses constraints to just suggest values to the user, or to optionally remove palely incompatible values (that is, values which, by inference, would produce an inconsistency in the model) from choice lists, and give in any case complete freedom to us$\operatorname{ers}^{10}$. Much the same way, when a property has been

\footnotetext{
${ }^{10}$ For example, when, by following a drag\&drop action, a value needs to be added to a resource, the range of suggested properties is first selected on those whose rdfs:domain is computed by inference to include at least one of (and be compatible with all of) the types of the subject resource.
} 


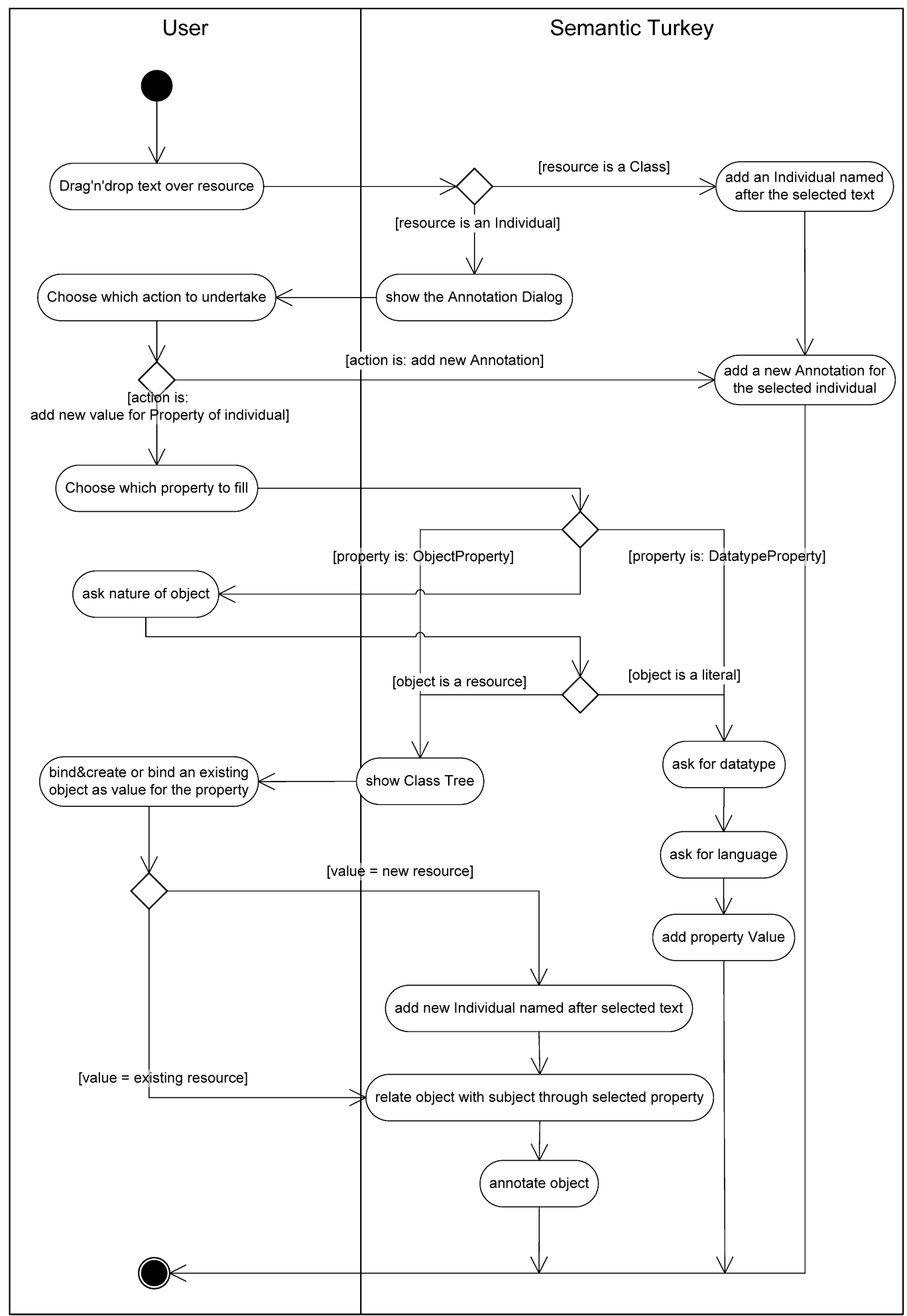

Fig. 3. Activity diagram for semantic bookmarking/annotation. 
selected for adding a value, resources can be selected from a class tree-view rooted on the rdfs:range of the property (with analogous considerations to the previous case). These suggestions can be bypassed (e.g. asking to display all the properties, or to explore the whole class tree instead of the suggested part), in that the user can go out of available boundaries, and introduce new "implicit" knowledge by adding ground facts which alter, by inference, the knowledge of the domain. This kind of interaction surpasses the limitations of (at least some of) current ontology editing tools, which are still not fully acquainted with the inferential aspects of the OWL language. For example, Protégé OWL 3.x [5], though offering advanced features and wizards for assisting users in adding entries to an ontology, is still bound to its original constraints-based model [4] which binds subject and object values of triples to the defined rdfs:domain and rdfs:rangeof the predicate.

Protégé $4^{11}$, being completely targeted for the Owl standard, abandons this constrained approach, though property-value editing is still in its infancy and, at present time, its authors preferred to not address at all classification-related issues and to show instead the (whole) list of available instances when the user asks for potential values to be added to object properties. Semantic Turkey thus makes ontology editing faster by proposing suggestions to the users, which rely on declared restrictions and on asserted (or inferred) types and values, but they can always break these boundaries and have access to the whole data, eventually letting further inference follow its actions.

\subsection{Other features}

Semantic Navigation. As an additional feature, the user may graphically explore the ontology, thanks to the Semantic Navigation component. A Java applet will be loaded on a new tab of the browser, displaying the graph view of the ontology, allowing the user to navigate its content. The nodes of the graph will be displayed in different manners, according to the nature of the ontological entity: classes, properties or individuals. By dragging the mouse pointer on a node that represents an individual, it is possible to popup a window, which contains the URLs of the pages where that instance has been annotated.

Extensibility. The drag'n'drop macros for ontology editing/annotation are just a nod to what can be done in a browser-embedded ontology editor: ST's

\footnotetext{
${ }^{11} \mathrm{http}: / /$ protege.stanford.edu/download/registered.html\#p4
}

flexible extension mechanism allows for dedicated extensions to be realized, exploiting different interaction possibilities with the user and making it possible to deliver completely new applications based on the Knowledge Management infrastructure of ST (see Section 6, 6.2 in particular).

\section{Knowledge model}

ST offers complete functionalities for importing ontological data coming from different RDF/OWL sources. Its internal Knowledge Model (KM) foresees a separation between the explicit (domain) knowledge managed by the user and the one which guides the system's behavior. This last layer, defined as the Application Ontologies Layer, is kept invisible to the user, and is only exploited by the application to drive it knowledge based functionalities. Semantic Turkey currently includes one vocabulary in this layer, the Annotation Ontology: a set of concepts (and related properties) used to keep track of annotations from the Web. These include:

- ann:WebPage (rdfs:subClassOf ann:Document) concept for storing information about the annotated pages (such as ann:URL and ann:title), that is, the pages where part of the text is annotated with respect to the ontology and thus added to it as a new individual

- ann:SemanticAnnotation containing the annotations performed by the user, and described by the bookmarked ann:WebPage, resource etc. these can be both ann:TextualAnnotation(s) (for text annotated from the web page) as well as ann:ImageAnnotation(s) (for future extensions with image media).

The textual annotations also keep track of the different possible lexical realizations (ann:text property) that a same object may have exposed into different web pages: they are not addressed as alternative labels for the resource, but are uniquely associated to that specific annotation, since they may also refer misspelled entries or other kind of references which the user may not want to associate to the targeted resource. The annotated text is used to retrieve the textual occurrence of the resource when the user gets back to the same page (a highlighter icon in the bottom will show the presence of previous annotations on a page and will allow the user to view them highlighted). 


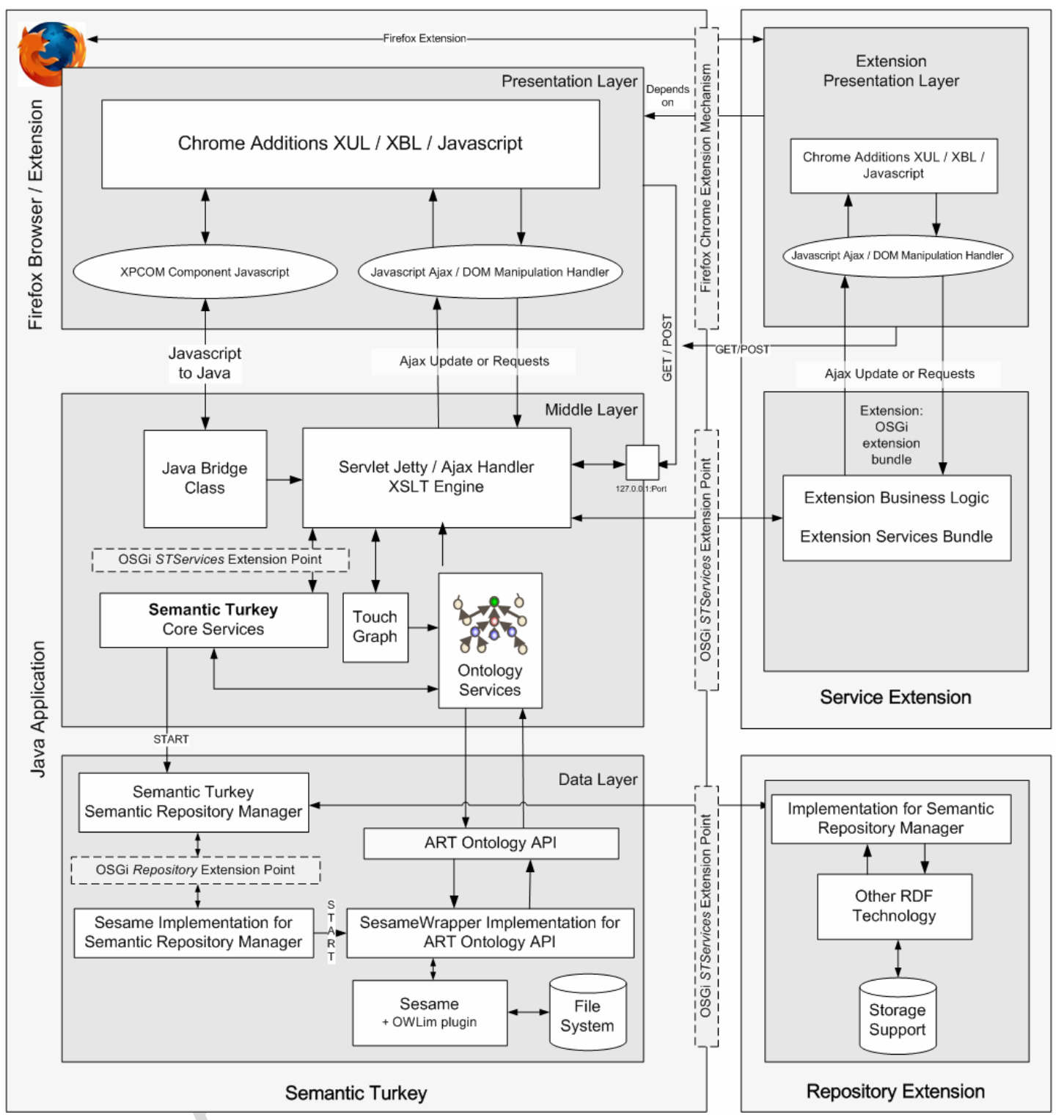

Fig. 4. Architecture of Semantic Turkey and of its extensions.

The Application Ontologies layer is not limited to include the sole Annotation Ontology, and can be dynamically extended to host new application ontologies according to the needs of Semantic Turkey extensions (see extension mechanism in the following section).

\section{Architecture}

The architecture (Fig. 4) of Semantic Turkey follows a three layered design, with the presentation layer embodying the true Firefox extension and the other two layers built around java technologies (also embedded in the extension) for administering the business logic and data access.

\subsection{Architectural layers}

The following paragraphs describe more in detail the three layers which constitute the architecture of Semantic Turkey

Presentation Layer. Everything relating user interaction is directly managed by the Firefox exten- 
sion. An $\mathrm{XPCOM}^{12}$ component has been developed to link the presentation layer to the service layer, which is implemented in java. This direct link is actually performed just to wake up an embedded Java web server, which accepts further messages from the client. This layer is actually not limited to presentation responsibilities, since much of the web-related processing (e.g. accessing pages, browsing their content, extracting portions of text etc.) can be delegated to the web scripting engine of the web browser.

Service Layer. This layer offers an extensible set of OSGi ${ }^{13}$ services which may be invoked through XMLHttpRequest(s), following the Ajax [25] paradigm. Besides supporting the communication with the client, the middle layer provides the functionalities for definition, management and treatment of the data and the business logic of applications built on top of Semantic Turkey framework.

Data Layer. It is mainly constituted by the component for managing the ontology. This has recently been rewritten as a series of dedicated middle-layer API for accessing ontological data: these offer both RDF triple-level access methods as well as more object-oriented tailored facilities, which have been appreciated in RDF libraries like Jena [26] (more details in the following section).

\subsection{The extension mechanism}

Semantic Turkey features an extension mechanism based on a combination of the Mozilla extension framework (which is used to extend the user interface, drive user interaction, add/modify application functionalities and provide javascript API for the whole set of Mozilla desktop utilities) and the OSGi java extension framework which provides extension capabilities for the service and data layers of the architecture.

OSGi compliance is obtained through the OSGi implementation developed by the Apache Software Foundation, called Felix ${ }^{14}$. Three main extension points have been introduced: a Service Extension, an Ontology Manager Extension and a Data Extension, to provide respectively: new functionalities, support for other data management technologies and for introducing new application ontologies.

Both the Java business logic layer and the Javascript layer for interaction with the browser pro-

\footnotetext{
${ }^{12} \mathrm{http} / /$ www.mozilla.org/projects/xpcom/

${ }^{13} \mathrm{http}: / /$ www.osgi.org/

${ }^{14} \mathrm{http}: / /$ felix.apache.org/
}

vide $\mathrm{API}^{15}$ for accessing/manipulating RDF data as well as for interacting with the core system and the browser hosting this application. Target users of this integrated development framework range from developers of web browser extensions willing to add RDF-based functionalities without the need to rewrite the whole infrastructure from scratch, to developers of knowledge acquisition tools, which get for free all the basic ontology management features and the possibility to interact with web content through a robust web browser (and its associated development environment).

\section{Comparison with state-of-the-art}

We have considered two recent test beds for evaluating Semantic Turkey with respect to state-of-the-art tools upon a functional comparison.

The SEALS (Semantic Evaluation At Large Scale) project $^{16}$ aims at facilitating the formal evaluation of semantic technologies. This allows both large-scale evaluation campaigns to be run (such as the International Evaluation Campaigns for Semantic Technologies) as well as ad-hoc evaluations by individuals or organizations. The evaluation campaign conducted in $2010^{17}$, covered:

1. Conformance of tools to languages of the RDF family (in the specific: RDFS, OWL 1 Lite, DL, Full).

2. Interoperability: how ontologies can be exchanged between different tools.

3. Scalability: the size of ontologies which can be loaded in these tools and the time needed to load them.

With respect to tools such as Protégé (both 3 and 4) and Neon Toolkit, which are based on API which do not work at RDF level (as reported in the "summary of the results") and expose thus some conformance and interoperability problem, the RDF abstraction layer of Semantic Turkey (the already cited OWL ART API) and its available implementations

\footnotetext{
${ }^{15}$ Interaction with the business logic of the system is provided by direct Semantic Turkey API, access to RDF is provided by OWL ART API (http://art.uniroma2.it/owlart/); the hosting browser is accessible through Mozilla Javascript language while STscript API (http://semanticturkey.uniroma2.it/documentation/ jsdoc) allow for browser side access to the service layer functionalities.

${ }^{16} \mathrm{http}: / /$ www.seals-project.eu/

${ }^{17} \mathrm{http}: / /$ www.seals-project.eu/seals-evaluation-campaigns/ ontology-engineering-tools/oet-2010-campaign-results
} 
(Sesame, Jena, AllegroGraph) is not prone to such issues. The sole limitation to interoperability (which would not emerge from SEALS tests) resides in OWL ART API (and Semantic Turkey as well) allowing for the creation of named resources identified by IRIs instead of restricting them to URIs (providing the backing API implementation allows for their creation, such in the case of Sesame2). This hampers compatibility with other tools (only in data export, still being able to virtually load any RDF conformant document), though it is actually more an advanced feature than a limitation, and could be easily restricted from the UI.

Starting from the functional comparison between ontology editing tools reported in [27], we also considered the functionalities exposed there (we refer the reader to the cited paper for more details) and remark those major pro and flaws with respect to tested applications:

- Ontology Storage: several options are available, depending on chosen triplestore. A menu dynamically produced during project creation ${ }^{18}$ allows for fine tuning of triple-store configuration depending on the chosen technology (e.g. activate reasoning, keep data in memory and save it on request or save it to mass-storage in realtime etc.). Surely a strong point of ST versus other considered tools (apart from Topbraid Composer ${ }^{19}$, which offers the same feature on Jena as an RDF middle-layer).

- Models: RDF, RDFS, OWL and $\mathrm{SKOS}^{20} \bmod -$ els; for SKOS: SKOS-XL support, multischeme management, etc., a gain over many available tools (possible exception is SKOSED, an extension available for Protégé 4).

- Inference: natively supported by API, specific reasoning depends on triplestore implementation.

- Collaboration: not available at the moment, just non-profiled concurrent access to same project.

\section{Conclusions}

In this paper we presented the latest release of Semantic Turkey: a semantic extension for the web browser providing functionalities for Knowledge

\footnotetext{
${ }^{18} \mathrm{http}: / /$ semanticturkey.uniroma2.it/documentation/projects.jsf\# createProject

${ }^{19}$ TBC was not considered in the cited paper because it is a review of non-commercial tools.

${ }^{20}$ The UI for SKOS is experimental in version 0.7.2.
}

Management and Acquisition. We discussed the main innovations introduced with respect to its original prototype and showed the potentialities of this framework by presenting its extension capabilities.

\subsection{Collected experiences and lessons learned}

The experiences that we have recently undergone in the adoption of Semantic Turkey across different application scenarios have been a test bed for evaluating the real possibilities of such an extensible framework. The result is that, though far from perfect, the extension mechanism (combining both open service gateways and browser interaction) is flexible enough to allow for very different uses of the platform. For example, the UIMAST [28] extension, developed in the context of the UIMA Innovation Award 2007 ${ }^{21}$, brings into ST the document analysis capabilities of the UIMA platform (a framework originally developed by IBM on top of the OASIS standard for Unstructured Information Management Architecture ${ }^{22}$, and lately devolved to the Apache Software Foundation), thus introducing functionalities for concept extraction from web pages and ontology learning.

UIMAST then allows users to literally interact through UI elements with the content of analyzed web pages.

ST extensions also range to totally new applications hosted on the web browser, which just rely on the underlying infrastructure for knowledge management. A success story in this sense is offered by STIA [29], an annotation environment for comparing web documents in the jurisprudence domain and for matching concepts from different laws, which completely hides underlying ontological details.

Developed inside our collaboration with the European Space Agency (ESA), in the context of their participation to the EU funded project Diligent (IST004260), EOAnnotator [30] is another extension showing how to exploit the browser to ease acquisition of contents from the web (in this case, through extraction and projection of RDFa from the browsed pages, over the ontology being edited by the user).

The above experiences also made us better understand the added value given by the underlying ontology development framework, which comprehends high level data access and manipulation primitives going far beyond basic RDF management, as it is commonly provided by triple store libraries/services such as Jena or Sesame.

\footnotetext{
${ }^{21} \mathrm{https}: / / \mathrm{www}-304 . i b m . c o m / j c t 09002 \mathrm{c} /$ university/scholars/innovation

$22 \mathrm{http}$ ://docs.oasis-open.org/uima/v1.0/uima-v1.0.html
} 
Finally, one more lesson gained from these experiences is that the learning curve for extension developers is a bit steep due to the wide range of employed technologies and to their different levels of integration: this will require even stronger attention on solutions and support for an Aided Extension Development, which goes beyond extensive documentation and probably embraces the realization of dedicated tools and development frameworks. Supporting the growth of a dedicated open software development community has been in fact one of the key aspects in several successful experiences (e.g. Protégé).

\subsection{User feedback}

We opened up tool evaluation to the user community through a questionnaire available at: http:// semanticturkey.uniroma2.it/questionnaire/.

Contributions are still few to trace a statistically significant analysis (also because the questionnaire provides different questions depending on the user profile, which may vary from "Domain Expert" to "Semantic Web Application Developer"), though we collected most prominent results (homogeneous across different users) which revealed Semantic Turkey's strong points and flaws:

- User interface is considered friendly. All voted from "satisfactory" to "yes, sure!" upon the explicit question about friendliness of $\mathrm{UI}^{23}$, and this has been remarked with comments - especially from domain experts - comparing it to other available tools.

- Easiness of installation is another strong point, though someone reported problems - in their comments to the answer - whenever Firefox java plugin is not properly setup: this is not something directly related to what can be done at application level, though we acknowledge that the underlying technology (java plugin, firefox xcom etc.) is not completely $100 \%$ guaranteed to work immediately on all machines, and may require some setup ${ }^{24}$.

- Extension Development: the very few users who completed this part (rating themselves as Se-

\footnotetext{
${ }^{23}$ Though 1) users of machines based on Mac OS experienced a few bugs due to idiosyncrasies in the Mozilla XUL language for UI description related to its Mac porting, and 2) the UI in general still has some flaws leaving room for improvement.

${ }^{24}$ These rare issues mostly affect Linux machines with nonSUN JVMs or not properly configured JVMs, thus happening to people with averagely more-than-average computer skills who know how to setup their system.
}

mantic Web Application Developers) rated the Extension Development learning curve as steep, thus confirming our considerations in previous section, though half of them really appreciated the possibilities of mixing different technologies and saw the learning phase as the necessary cost to pay for getting to them.

- Semantic Bookmarking and Annotation: the bookmarking feature of ST is seen as an added value with respect to existing tools: again, domain experts with no high computer skills provided most of the positive feedback. However, some of the users complained about lack of other bookmarking possibilities, such as bookmarking concepts other than instances: this feature has been requested to us especially by researchers working on Semantic Annotation who need to provide training datasets of pages tagged with respect to both entities and concepts.

\subsection{Future research work}

The next step which further development on this platform should take is to address the potentialities which have arisen by opening it up to full ontology development. In its new incarnation as a platform for development and acquisition of semantic web data, we cannot ignore important modeling axioms provided by the OWL language (restrictions, set operators etc. which are currently not available for editing, though being properly processed by the data\& inference layer), and include explicit support for different modeling frameworks, such as SKOS ${ }^{25}$.

On the other hand, while the above aspects are important in ontology development systems, there are other directions that, being by far more concerned with the contradistinguishing features of ST, could be properly investigated to push forward state-of-art research on this kind of framework. The presented architecture, thanks both to its modularity and web interaction features, could be lifted to a collaborative framework allowing knowledge engineers and domain experts to exchange information, opinions and data over the same working environment. Identification of proper user roles in the acquisition and development process could then give raise to a whole range of dedicated services being activated/hidden depending on the profile of the logged user. We are

\footnotetext{
${ }^{25}$ Both these features are currently being introduced in the platform. In particular, SKOS/SKOS-XL editing will be available in the next version to be released before end of 2010 .
} 
currently pursuing this objective [31], by introducing concepts close to (and inherited from) traditional solutions in Software Engineering: Bug-Tracking and Discussion, Issues Management, Versioning etc.

Another research line which naturally follows from the intrinsic connection between ontology and documents in ST, is related to the elicitation of knowledge from (web) resources: we are studying processes for automatically extracting knowledge from documents proactively collaborating with the user on how to use the collected information for populating/enriching managed ontologies (as for already cited UIMAST).

Finally, we found many overlapping points with current research on Semantic Desktops, especially in those modeling aspects which have been widely discussed and synthesized in the PIMO Ontology for Personal Information Models [32]. Interaction with this research field could be two-ways: by exploring assessed results in Semantic Desktop research, to better handle knowledge organization inside the current platform (e.g. by reusing PIMO ontologies in place of current annotation ontology), as well as by transforming ST into a browser end-point for Semantic Desktop interaction.

Semantic Turkey site (which reached now roughly 2700 downloads) can be reached at: http:// semanticturkey.uniroma2.it/.

\section{References}

[1] E. Seaborne, A. Prud'hommeaux. (2008, January) World Wide Web Consortium - Web Standards. [Online]. http://www.w3.org/TR/rdf-sparql-query/.

[2] C. Bizer, T. Heath and T. Berners-Lee, "Linked data - The story so far," International Journal on Semantic Web and Information Systems (IJSWIS), vol. 5, no. 3, pp. 1-22, 2009.

[3] D. Griesi, M.T. Pazienza and A. Stellato, "Semantic Turkey - A semantic bookmarking tool (system description)," in The Semantic Web: Research and Applications, 4th European Semantic Web Conference, ESWC 2007, Innsbruck, Austria, June 3-7, 2007, Proceedings. Lecture Notes in Computer Science, vol. 4519, 2007, pp. 779-788, Innsbruck, Austria, June 3-7.

[4] J. Gennari et al., "The evolution of Protégé-2000: An environment for knowledge-based systems development," International Journal of Human-Computer Studies, vol. 58, no. 1, pp. 89-123, 2003.

[5] H. Knublauch, R.W. Fergerson, N.F. Noy and M.A. Musen, "The Protégé OWL plugin: An open development environment for semantic web applications," in Third International Semantic Web Conference - ISWC 2004, Hiroshima, Japan, 2004.

[6] P. Haase et al., "The NeOn ontology engineering Toolkit," in $W W W 2008$ Developers Track, April, 2008.

[7] D. Quan and D. Karger, "How to make a semantic web browser," in Thirteenth International World Wide Web
Conference (WWW2004), New York City, USA, May, 2004.

[8] M. Dzbor, J. Domingue and E. Motta, "Magpie: Towards a semantic web browser," in 2nd International Semantic Web Conference (ISWC03), Florida, USA, 2003.

[9] M. Dzbor, E. Motta and J.B. Domingue, "Opening up Magpie via semantic services," in 3rd Intl. Semantic Web Conference (ISWC04), Hiroshima, Japan, 2004.

[10] T. Berners-Lee, J.A. Hendler and O. Lassila, "The Semantic Web: A new form of Web content that is meaningful to computers will unleash a revolution of new possibilities," Scientific American, vol. 279, no. 5, pp. 34-43, 2001.

[11] D. Huynh, S. Mazzocchi and D.R. Karger, "Piggy bank: Experience the semantic web inside your web browser," in Fourth International Semantic Web Conference (ISWC05), Galway, Ireland, November, 2005, pp. 413-430.

[12] T. Berners-Lee, Y. Chen, L. Chilton, D. Connolly and R. Dhanaraj, "Tabulator: Exploring and analyzing linked data on the semantic web," in 3rd International Semantic Web User Interaction Workshop in International Semantic Web Conference, Athens, Georgia, USA, 2006.

[13] J. Kahan and M.-R. Koivunen, "Annotea: An open RDF infrastructure for shared Web annotations," in $W W W^{\prime} 01$. Proceedings of the 10th international conference on World Wide Web, Hong Kong, Hong Kong, 2001, pp. 623-632.

[14] V. Quint and I. Vatton, "Techniques for authoring complex XML documents," in DocEng 2004, ACM Symposium on Document Engineering, 2004

[15] M. Wilson. http://annozilla.mozdev.org/.

[16] F. Ciravegna, A. Dingli, D. Petrelli and Y. Wilks, "Usersystem cooperation in document annotation based on information extraction," in 13th International Conf. on Knowledge Engineering and Knowledge Management, EKAW02, 2002.

[17] B. Popov et al., "KIM semantic annotation platform," in 2nd International Semantic Web Conference (ISWC2003), vol. 2870, Florida, USA, 2003, pp. 834-849, 20-23 October.

[18] Chakravarthy, F. Ciravegna and V. Lanfranchi, "AKTiveMedia: Cross-media document annotation and enrichment," in Poster Proceedings of the Fifteenth International Semantic Web Conference (ISWC2006), 2006.

[19] V. Uren et al., "Semantic annotation for knowledge management: Requirements and a survey of the state of the art," Journal of Web Semantics, vol. 4, no. 1, pp. 14-28, 2006.

[20] D. Griesi, M.T. Pazienza and A. Stellato, "Gobbleing [sic] over the Web with Semantic Turkey," in Semantic Web Applications and Perspectives, 3rd Italian Semantic Web Workshop (SWAP2006), ScuolaNormaleSuperiore, Pisa, Italy, 2006, 18-20 December.

[21] Dingli, F. Ciravegna and Y. Wilks, "Automatic semantic annotation using unsupervised information extraction and integration," in Workshop on Knowledge Markup and Semantic Annotation (K-CAP), 2003.

[22] J. Euzenat, "Eight questions about semantic web annotations," IEEE Intelligent Systems, vol. 17, no. 2, pp. 55-62, 2002 .

[23] J. Broekstra, A. Kampman and F. van Harmelen, "Sesame: A generic architecture for storing and querying RDF and RDF schema," in The Semantic Web - ISWC 2002: First International Semantic Web Conference, Sardinia, Italy, 2002, pp. 54-68, June 9-12.

[24] Kiryakov, D. Ognyanov and D. Manov, "OWLIM - A pragmatic semantic repository for OWL," in Int. Workshop on Scalable Semantic Web Knowledge Base Systems (SSWS 
2005), WISE 2005, New York City, USA, 2005, 20 November.

[25] J.J. Garrett. (2005, February) Ajax: A New Approach to Web Applications. [Online]. http://www.adaptivepath.com/ publications/essays/archives/000385.php.

[26] B. McBride, "Jena: Implementing the RDF model and syntax specification," in Semantic Web Workshop, WWW2001, 2001.

[27] B. Kapoor and S. Sharma, "A comparative study ontology building tools for semantic web applications," International Journal of Web \& Semantic Technology (IJWesT), vol. I, no. 3, July 2010

[28] M. Fiorelli, M.T. Pazienza, S. Petruzza, A. Stellato and A. Turbati, "Computer-aided ontology development: An integrated environment," in New Challenges for NLP Frameworks 2010 (held jointly with LREC2010), La Valletta, Malta, 2010, 22 May.

[29] M.T. Pazienza, N. Scarpato and A. Stellato, "STIA: Experience of semantic annotation in jurisprudence domain," in Proceeding of the 2009 Conference on Legal Knowledge and Information Systems, vol. 205, 2009, pp. 156-161.
[30] F. Fallucchi et al., "Semantic bookmarking and search in the earth observation," in Knowledge-Based Intelligent Information and Engineering Systems. 12th International Conference, KES 2008, Zagreb, Croatia, September 3-5, 2008. Lecture Notes in Computer Science, vol. 5179/2008, 2008, pp. 260-268, mine.

[31] D. Bagni, M. Cappella, A. Stellato and M.T. Pazienza, "CONGAS: A COllaborative ontology development framework based on Named GrAphS," in International Conference of the Italian Association for Artificial Intelligence (AI*IA09), Reggio Emilia, Italy, 2009, December, 9-11.

[32] L. Sauermann, L. van Elst and A. Dengel, "PIMO - A framework for representing personal information models," in Proceedings of I-MEDIA'07 and I-SEMANTICS'07 International Conferences on New Media Technology and Semantic Systems as part of (TRIPLE-I 2007), Graz, Austria, September 5-7, 2007.

[33] S. Braun, V. Zacharias and H.-J. Happel, "Social semantic bookmarking," in Practical Aspects of Knowledge Management, T. Yamaguchi, Ed.: Springer Berlin/Heidelberg, 2008 , vol. 5345, pp. 62-73, 10.1007/978-3-540-89447-6 8 . 